\title{
Fallopian Tube Carcinoma
}

\author{
Agrawal Shalini $\cdot$ Gupta Taru
}

Received: 26 September 2009/ Accepted: 28 May 2012/Published online: 12 March 2013

(C) Federation of Obstetric \& Gynecological Societies of India 2013

\section{Introduction}

Primary adenocarcinoma of the fallopian tube is a rare gynecological malignancy affecting females in the fifth and sixth decade of their lives. The incidence varies from 0.16 to $1.6 \%$ with an average of $0.3 \%$. The rarity of such types of carcinoma mandate us to report it as an individual case.

\section{Case Report}

A 60-year-old multiparous postmenopausal woman was admitted with the complaint of pain in the lower abdomen for the past 2 months. Clinical and relevant investigations revealed a left-sided adnexal mass of suspected ovarian origin with solid and cystic components with mild ascites. Tumor markers were assayed. CEA was raised $11.69 \mathrm{ng} / \mathrm{ml}$ $(N<2.5)$, CA-125 was $39.9 \mathrm{U} / \mathrm{ml}(N<35)$, and AFP was normal. Colonoscopy was done and was normal. With findings of a left adnexal mass on CECT and marginally raised tumor markers, the patient was counseled and accepted for exploratory laparotomy and surgical staging. Minimal straw-colored ascitic fluid was present, which was

Agrawal S., Senior Consultant · Gupta T., Consultant Department of Gynaecology and Obstetrics, Maharaja Agrasen Hospital, New Delhi, India

Agrawal S. ( $₫)$, Senior Consultant A1/17, GF, Janakpuri, New Delhi 110058, India e-mail: drshalini57@gmail.com saved for cytology for malignant cells and a PCR test for Tubercle bacilli. Grossly left adnexa showed an encapsulated solid mass in the ampullary portion of the left tube measuring $5 \times 3 \times 3 \mathrm{~cm}$. The left tube was identified by fimbriae at the lateral end of the mass. Both ovaries were atrophied. The omentum looked normal in appearance and texture. The parietal and visceral peritoneum looked grossly normal. Total abdominal hysterectomy with bilateral salpingo oophorectomy, bilateral pelvic lymph node sampling, and omental biopsy was done. A biopsy from a suspicious nodule on the round ligament was taken. A cut section of the mass showed solid and cystic areas filled with clear fluid. A microscopic section through the solid left tubal mass showed the fallopian tube with a tumor composed of round to oval cells with enlarged pleomorphic vesicular nuclei and eosinophilic cytoplasm arranged in a glandular pattern as well as in cords and clusters. The formation of papillae and cribriform pattern along with areas of coagulation, necrosis and mitotic activity were seen. The stroma showed mixed cell infiltration and the tumor was found invading the full thickness of the wall of the tube and reaching up to the serosa. The fimbrial end was uninvolved. The report was suggestive of mixed surface epithelial adenocarcinoma (endometrioid carcinoma $60 \%$ and serous papillary carcinoma $40 \%$ ) of the left fallopian tube, grade 2, invading the muscularis propria, reaching up to the serosa. There was no angio-lymphatic invasion seen. The uterus, cervix, vaginal flaps, and both ovaries were negative for malignancy. Ascitic fluid, omental biopsy, and pelvic lymph nodes were free from the 
tumor. The patient was advised chemotherapy (carboplatin and docetaxel) for six cycles, but she did not come for follow-up.

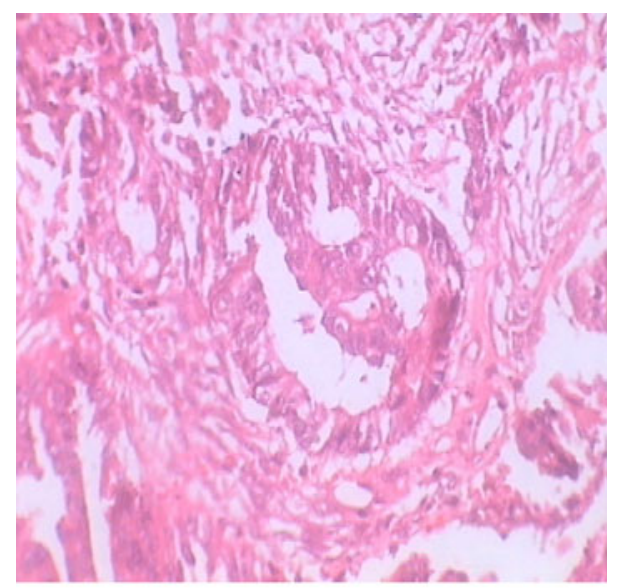

Acinar Differentiation

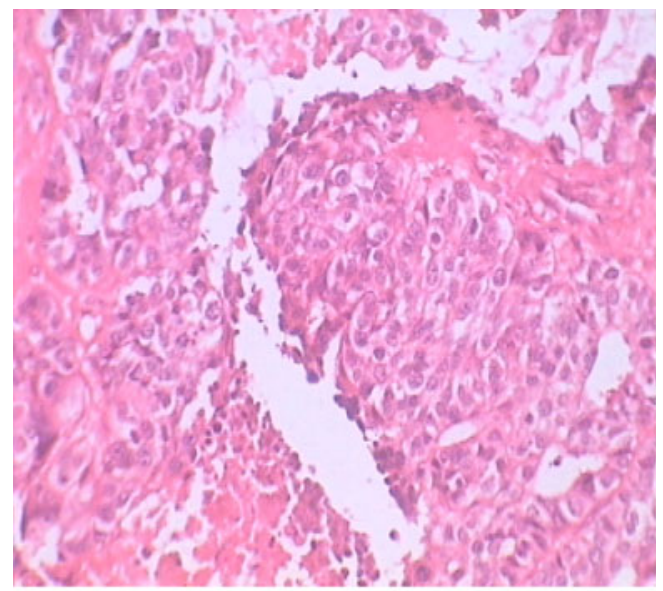

High Power Adenoca changes in the adjacent mucosa and the uterus and the ovaries should be normal and completely separate from the tumor. The presenting symptoms are variable and nonspecific with pre-operative diagnosis rarely entertained. The FIGO system for staging fallopian tube carcinoma is similar to the surgical staging criteria for ovarian carcinoma. Unlike ovarian tumors, more than $50 \%$ of fallopian tube tumors are in stages 1 and 2 with a predilection for metastasis to retroperitoneal lymph nodes in contrast to the intraperitoneal spread of ovarian carcinoma.

Fallopian tube cancer is the least common of gynecological malignancies [1]. The mean age of presentation is quoted as 56 years with most cases presenting in the fifth and sixth decade. It is more common in postmenopausal women. Our patient was also a 60-year-old postmenopausal woman, comparable to other studies. The classic triad of symptoms, a serosanguinous discharge, pain and an adnexal mass, and hydrops tubae profluens, as described by Latzko in 1916 is rarely reported. The presenting symptoms are non-specific. Patients with abdominal pain seek medical attention much earlier than those with vaginal discharge or menstrual problems. The pain is colicky and lower abdominal in nature, which may be related to distension of a partially blocked fallopian tube by fluid, which is then relieved by the passage of blood or discharge. Thus, these clinical features were comparable to other studies [2].

Owing to its rarity, stringent diagnostic criteria have to be adapted [3]:

(a) Main tumor mass to be confined to the tube with demonstrable carcinoma in situ changes in the adjacent mucosa.

(b) Uterus and ovaries should be normal.

These criteria were fulfilled in our case.

\section{References}

\section{Discussion}

In 1847, Renaud first described fallopian tube malignancy. In 1888, Orthmann submitted the first genuine case report. For the diagnosis to be made, the main tumor mass must be confined to the tube with demonstrable carcinoma in situ
1. Hanton EM, Malkasian GD Jr, Dahlin DC, et al. Primary carcinoma of the fallopian tube. Am J Obstet Gynecol. 1996;94:832-9.

2. Ng P, Lawton F. Fallopian tube carcinoma-a review. Ann Acad Med Singap. 1998;27:693-7.

3. Yogiraj SS, Vasantrao KP, Ranganthrao SB. Fallopian tube carcinoma. Indian J Pathol Microbiol. 2008;51:261-2. 